\title{
The JISH speech, language, and hearing school readiness screening in jeddah, saudi arabia
}

\begin{abstract}
This retrospective study presents the results of an Arabic school readiness screening conducted between the years of 2000 and 2010 at the Jeddah Institute for Speech and Hearing (JISH) in Jeddah, Saudi Arabia. The screening was requested by certain schools in the city of Jeddah and Makkah to investigate the readiness of children before entering school to make sure these children do not exhibit speech, language or hearing issues that might interfere with their academic performance and success at school in the first grade. A total of 1543 (1349 boys and 194 girls) screening records were reviewed and analyzed. The mean age of the children was 5; 09 years; months. The JISH Arabic Speech, Language, and Hearing Screening Protocol were administered individually by ASHA-certified speech-language pathologists. Analysis of results showed statistically significant prevalence rates in all areas for which screening was conducted: hearing $(7.1 \%$ " 0.0000 "); language comprehension $(1.7 \%$ " 0.0000$)$; language expression (2.7\% " 0.0000$)$; voice $(5.1 \%$ " 0.0000$)$; fluency $(3.2 \%$ " 0.0000$)$, and articulation $(30.3 \%)$. The findings of the current study assure the need to screen speech, language and hearing skills of children before sending them to schools. This is significantly needed in the Middle East and in the whole Arab region due to the fact that general physical examinations are not enforced in most of the medical insurance policies, therefore, communication deficits will not be detected from early ages.
\end{abstract}

Keywords: speech, language, hearing, school, screening, readiness, Jeddah, academic, prevalence, risk
Volume 7 Issue 5 - 2017

\section{Yaser Numan Al Sabi}

Speech Language and Hearing Science Department Dar Al Hekma University Jeddah, Saudi Arabia

Correspondence: Yaser Numan AI Sabi Assistant Professor Speech Language and Hearing Science Department Hekma School of Education and Health Sciences Dar Al Hekma University Jeddah, Saudi Arabia, Tel 0096650826 1992, Email yasersab2002@yahoo.com

Received: July 25, 2016 | Published: June 20, 2017
Abbreviations: JISH, jeddah institute for speech and hearing; ASHA, american speech-language-hearing association; OSMSE-R, oral-speech-mechanism-screening-examination-revised; $\mathrm{dB}$, decible; $\mathrm{HL}$, hearing level; $\mathrm{Hz}$, hertz; $\mathrm{COHC}$, certified occupational hearing conservationist; AAA, american academy of audiology; AA, african american; UN, united nations; WHO, world health organization; SADAFCO, saudia dairy and foodstuff co

\section{Introduction}

Children exhibiting speech and language deficits are at a great risk for academic, learning, behavioral, and social problems. ${ }^{1}$ Children's communication skills play a major role in their early success in life at all levels: cognitive, social, emotional, personal, and academic among these. Academic skills - reading and writing, for example, are dependent on an adequate sound system. Dyslexia is a disorder that affects the student's ability to develop reading skills. According to Bender" "there is a least one other possible cause of dyslexiathe language or encoding capability of the individual with learning disabilities (p. 162). It is of great importance, therefore, to assure that a young child's speech and language skills are adequate to proceed and succeed in childhood development and future life learning.

Prevalence studies are one way of gathering data on the percentage of children affected with a certain disorder or deficit. Prevalence refers to the number of, or proportion of a population that is affected by a condition at a given point in time (http://www.asha.org/members/ ebp/Glossary/\#P, retrieved June 20, 2016, 12:45 PM). ${ }^{3}$ Prevalence studies have been conducted that estimate the amount of children who demonstrate speech and language delays and other communication issues in several countries and regions in the world, including western/ European countries (United States of America, Belgium) and (Turkey and Greece)-(McLeoud \& Harrison, 2009). ${ }^{4}$ The United
States Prevalence Task Force $(2006)^{5}$ estimates the prevalence of speech and language delays of preschool children is between 5 $8 \%$. In a single prevalence study examining the prevalence and risk factors for hearing loss in school-aged children in Egypt, Taha et al., ${ }^{6}$ estimated the prevalence of hearing loss among school children at $20.9 \%$. El-Zraigat ${ }^{7}$ reported that hearing loss disability is the most second prevalent disability in Jordan. In a study conducted by the Jordanian Ministry of Health, and the World Health Organization in 2005 , to investigate the incidence, prevalence, risk factors and causes of hearing loss in Jordan, six thousand participants ranging in ages from 6months to 60years were selected from 8 governorates in Jordan (Amman, Irbid, Zarqa, Mafraq, Jerash, Ajlun, Madaba, and Balqa). Forty four percent of the participants were males. The results of the study revealed that the prevalence of hearing loss (HL) in Jordan was $8.2 \%$, which is five times more than that in the developed countries $(0.8 \%){ }^{8}$ These studies note the relatively high prevalence and increased risk for these disorders among young children. Unfortunately to date, few prevalence studies of speech-language disorders have been conducted in the Arab Region. The absence of prevalence data impacts the provision of adequate services, as adequate planning on a systematic level within a region relies on prevalence data. ${ }^{9}$

Screening of children for speech-language or hearing disorders is one of the major clinical services provided by a speech-language pathologist. A screening is a brief assessment conducted by a professional and aims at identifying those individuals who are at risk for or may be demonstrating developmental delays. ${ }^{10}$ Screening is often done with infants and young children to identify those disorders that are found at a higher prevalence in a population or at a point in life where specific disorders are at a high risk for occurring, as in the case of speech, language, and hearing issues among young children. When communication delays are detected and addressed early, chances for improvement are significantly better. ${ }^{11,12}$ 
Unfortunately, speech-language and hearing screenings conducted in the past two decades have been questionable with regards to many issues related to the quality of results gathered and presented, including the reliability and validity of screening tools, the qualifications of testers and examiners, the methodologies used during screening (indirect screening methods such as parent and teacher questionnaire versus direct testing) and the sampling representativeness. ${ }^{13-20}$ These limitations call into question the validity of the findings of prevalence studies, given the absence of trained staff conducting many of the screenings, the lack of standardized methods used in these screenings, and the lack of reliability between raters using these tools ${ }^{21}$ and call into conclusive answers for the optimal ages and for the frequency of screenings. ${ }^{22}$

This study addresses the above noted limitations and gaps in the research on speech, language and hearing disorders in young children in the Arab Region. It introduces a screening tool developed to identify speech, language, and hearing disorders among young, Arabicspeaking children. It provides potentially valuable information to Arabic speaking speech-language pathologists, parents and teachers regarding the prevalence of delays or disorders in the areas of speech, language, and hearing that can assist in guiding effective intervention. Further, it provides potentially critical information to policy-makers in the Ministries of Education and Health in the Kingdom of Saudi Arabia and across the whole Arab Region. Attention was also paid to the methodological limitations noted in existing prevalence studies. This study did not rely on indirect assessment methods, such as the sole use of parent or teacher questionnaires; all participants were directly tested on skills included on the screening tool. Further, all screenings were conducted by qualified, ASHA-certified speechlanguage pathologists who possessed a minimum of a master's degree in speech-language pathology and audiology and who possessed a minimum of five years of experience conducting screening and speech-language and hearing assessment.

Specifically, the present study was conducted to achieve the following goals:

i. To obtain estimates of prevalence of speech, language, and communication disorders and hearing disorders among Arabicspeaking children in the Kingdom of Saudi Arabia prior to entering school (in order to assist in determining the readiness of these children for school);

ii. To determine the types of speech and language deficits exhibited among Arabic-speaking children, in order to assist in educational and therapeutic planning;

iii. To categorize the types of speech and language deficits and disorders found among Arabic-speaking children in terms of severity and frequency;

iv. To compare the estimated prevalence rates of various speech and language deficits and disorders with the findings available in other prevalence studies;

v. To describe and highlight the role of the speech-language pathologist in facilitating academic skills (reading and writing) and the need to increase the recruitment and use of speechlanguage professionals in schools settings in the Arab Region;

vi. To provide the Arabic-speaking speech language pathologist and other professionals with necessary information, tools, and procedures to guide in the conducting of screening children prior to entering school; vii. To present the JISH Arabic Speech, Language, and Hearing Screening Protocol;

viii. To establish a preliminary estimate of speech, hearing, and language deficits and disorders among Arabic-speaking preschoolers in the Kingdom of Saudi Arabia.

\section{Materials and methods}

The records of 1543 children were who were screened using the JISH Arabic Speech, Language and Hearing Screening Protocol between the years of 2000 and 2010 were reviewed. These children received these screenings either at a private center located in Jeddah, Saudi Arabia, not affiliated with the developer of the screening protocol, or at JISH center itself. The children were referred for the screening from several schools within the city of Jeddah and surrounding regions, including: Al Manarat School, Daar Al Hanaan School, Al Bir Society for Orphanage School, and Jeddah Knowledge School, located in the city of Jeddah, Saudi Arabia and Al Falaah School, located in the city of Mecca Al Mukarrama, Saudi Arabia. Each of these schools required screening annually for all new enrollments, regardless of level or ability. The children's ages ranged between 4 years and 6years, 10 months. The majority of participants $(950$, or $61 \%$ of all participants) were between the ages 5years, 9 months and $6 y e a r s, 10$ months age-range. Three hundred and fifty seven children ( $23 \%$ of all participants) were between the ages 6years, 4 months and 6years, 10months; two hundred and twenty five children (15\%) were between the ages 5 years, 2 months and 5years, 8 months; nine children ( $0.6 \%$ of all participants) were between the ages 4years, 7 months and 5years, 1 month, and two children only ( $0.1 \%$ of all participants) were less than 4years, 7 months (Tables $1 \& 2$ ). The review of the records indicated that the vast majority of the children who participated in the screening came from Al Falaah School, Daar Al Hanaan School, and Al Manarat School consecutively. One thousand, three hundred and forty nine children who participated in the screenings were boys (87.4\%) and 194 were girls (12.6\%).

\section{Setting}

This data analysis portion of this study was conducted at the Jeddah Institute for Speech and Hearing (JISH), ${ }^{23}$ located in Jeddah, Saudi Arabia. The records reviewed for this study were all stored and maintained by JISH, and all record reviews were done on JISH premises. JISH is a not-for-profit institution established in 1991 as the first institute entirely devoted to speech-language and hearing service delivery in Saudi Arabia.

From its inception, JISH adopted the standards of clinical practice of the American Speech and Hearing Association (ASHA) to guide its supervision, training of staff, and service delivery. The screenings were conducted in Jeddah Institute for Speech and Hearing (JISH) and at a second center in the same city, by prior agreement between the two organizations.

\section{Materials}

The following materials were used to conduct each screening: The JISH Arabic Speech-Language-Hearing-Screening Protocol (described below), screening record (pass-fail forms) and the OralSpeech-Mechanism-Screening-Examination-Revised (OSMSE-R). The OSME- $\mathrm{R}$ is a standardized measure of the anatomical structures and psychological functions that are most often considered to be potentially related to speech or language disorders. It was designed as a screening tool for speech language pathologists, and is written in English. Other materials used during each screening included: 
gloves, tongue depressors, penlight, stickers, and an audiometer. The following types of audiometers were used for the hearing screenings: GSI 17 Portable Audiometer, manufactured by Lucas-Grason-Stadler, Inc, located in Littleton, MA (USA); SD21 (type 4), manufactured by DANPLEX A1S, 1302, Copenhagen K, Denmark; and GSI Clinical Audiometer (Class 1, Type B), manufactured by Grason-Stadler, Milford, NH (USA). The first two audiometers were used for the screenings conducted between the years 2000 and 2007, and the last audiometer was used from 2008 onward.

\section{The JISH Arabic speech-language-hearing-screening protocol}

This protocol was developed and internally validated by a group of Arabic speaking ASHA-certified speech-language pathologists employed by JISH. The screening protocol consists of an examiner's book and scoring sheet. The protocol is designed to cover the following sections: hearing, behavioral observations, language comprehension, language expression, sentence repetition, articulation, voice, and fluency. Items included in each section were selected by a team of speech language pathologists based on research dictating developmental norms, and personal experiences as well as on similar tools prepared in other languages, such as the Fluharty Preschool Speech and Language Screening Test. ${ }^{24}$

The JISH Arabic Speech, Language, and Hearing Screening Protocol consist of the following subtests:

a. Responding to general questions, such as "what is your name?", "How old are you?" and "What is your favorite game?";

b. Identifying 30 picture cards depicting nouns, verbs, adjectives, etc. The words were selected and agreed upon based on the clinical experience and observation of all the speech-language pathologists who participated in the development of the protocol;

c. Following auditory or spoken directions, divided into three subsections in the following order: following one-step directions, following two-step directions, and finally following three-step directions; (4) naming 20 picture cards;

d. story telling using six sequenced cards depicting a boy falling off his bike; Pictures and objects that are culturally appropriate were used in the screening protocol;

e. Comprehension questions: this subtest consists of seven questions on the story presented in the previous subtest. Different types of questions are used, i.e., yes-no questions and wh-questions;

f. Articulation subtest: this subtest screens all 28 Arabic sounds in the word position. Ten sentences were selected and agreed upon by developers for the articulation subtest. Sentences vary in length and complexity;

g. fluency section is designed to informally screen fluency skills of participant to make sure that the child does not exhibit any fluency problems that warrant further evaluation; and

h. Voice, resonance and prosody subtest is designed to perceptually screens the voice, prosody and resonance skills and make sure that these skills are age-appropriate.

To assure reliability, test-retest reliability was calculated. Test-retest is defined as "the consistency of a measure from one administration to another". ${ }^{25}$ The screening was administered twice over a two-week interval by same examiner. Pearson's correlation coefficient indicated no statistical difference between the test and the retest scores ( $r .094$ at a significance level of $\mathrm{P} \leq 0.01)$. The results of the reliability coefficient indicated no differences in the results of the participants when tested twice within 7 to 14 days.

Results in Table 3 below show that the reliability coefficients for all of the sections of the screening ranged between 0.92 and 0.98 , indicating strong reliability. Content validity of the screening was also examined and judged by four independent speech-language pathologists who are experienced in test development and speechlanguage testing. All four speech-language pathologists agreed that the screening items were adequate and representative of the domains the screening was designed for. It was not possible to test the criterion-related validity of the JISH Screening due to unavailability of other screening protocols in Arabic at the time when the JISH Screening protocol was developed. The construct validity of the JISH Arabic Speech-Language-Hearing-Screening Protocol was also used to examine the effectiveness of the protocol in screening what the protocol is designed for. Construct validity is concerned with the degree the instrument measure the theoretical construct for which it is designed to measure (e.g., receptive language). As per the Standards for Educational and Psychological Testing, construct validity is achieved through extensive research studies. ${ }^{26}$ The results indicated high level of construct validity.

\section{Procedure}

The JISH Speech-Language-Hearing Screening Protocol was used to screen the communication skills and hearing acuity of the identified participants. The hearing screening was conducted initially prior to the speech-language screening. ASHA hearing screening guidelines were followed and adopted by an audiologist employed by JISH. Every child was screened at fixed intensity of $20 \mathrm{~dB}$ HL at 1000 , 2000 and $4000 \mathrm{~Hz}$. The speech-language screening was conducted individually for every child by an ASHA-certified speech-language pathologist employed by JISH. Results and recommendations of the screenings were provided to the parents and the schools. Possible recommendations included further evaluation in a specific area, comprehensive evaluation, re-screening, or no further follow-up.

\section{The Speech-language pass-fail criteria}

The JISH Speech-Language-Hearing Screening Protocol has not been standardized; consequently, the performance of the participants was recorded using a pass-fail criterion. The pass-criterion level was agreed upon by the consensus of the examiners as well as the developers of the protocol. The pass-fail criterion for each section of the protocol is described below:

For the language comprehension section, the performance of the participant was recorded as pass if he/she identified at least $80 \%$ of the targeted picture cards (i.e., at least 24 out of the 30 stimuli items) correctly; For the following directions section, the performance of the participant was recorded as pass if he/ she followed $80 \%$ of given directions. This section was considered as part of the language comprehension section and the criteria was the same; For the language expression section, the performance of the participant is recorded as pass if he/she named a minimum of $80 \%$ of the targeted picture cards (i.e., at least 16 out of the 20 stimuli items). For the articulation section, the performance of the participant was recorded as pass if he/she did not exhibit any articulation error. Errors that are developmental in nature were not considered in the fail criterion. For fluency section, the pass criterion was recorded if the participant did not exhibit episodes of dysfluencies, namely, part-word repetitions, prolongations, interjections, and pauses. However, if the participant 
obtained a score of 0 on at least 3 of the core behaviors of stuttering (part-word repetition, prolongations, interjections, and pauses), and with at least $5 \%$ of dysfluencies from the speech sample obtained, then he/she will be considered fail.

Finally, for the voice section, the participant's performance was recorded perceptually by the examiner. If the participant was perceptually judged by the examiner to exhibit a voice characterized by one of the following characteristics, then he/she will be judged as fail: breathiness, harshness, hoarseness, or huskiness. Otherwise, the participant will be given pass.

\section{Examiners}

As per the American Speech and Hearing Association's (ASHA's) fundamental standards and guiding principles, the screenings were conducted by certified and credentialed speech-language pathologists who have extensive experience in screenings and testing ${ }^{27}$ and who all hold Master's Degree in speech-language pathology and audiology and the Certificate of Clinical Competence (CCC) from the ASHA The hearing screening was conducted by a Certified Occupational Hearing Conservationist (COHC), who works under the supervision of a clinical audiologist, holding a certificate of clinical competence in audiology from ASHA and board certified in audiology from the American Academy of Audiology (AAA). It is important to mention here that the same certified occupational hearing conservationist was in charge of all the hearing screenings conducted for the participants of this study.

\section{Hearing screening}

All hearing screenings were conducted in a manner congruent with appropriate infection control and universal precaution requirements at the Jeddah Institute for Speech and Hearing (JISH). ${ }^{28}$ Hearing screenings were conducted under earphones using, 1, 2, and $\mathrm{KHz}$ tones at $20 \mathrm{~dB}$ HL. The hearing screening results were scored on pass/ fail criteria. Each participant was judged as pass if his/her responses were judged to be clinically reliable at each frequency in each ear.

However, if the participant did not respond at any frequency in either ear, the examiner will reinstruct, reposition insert earphones, and rescreen the participant within the same screening session. If the participant did not respond following the repeated screening, then the participant was judged as fail and would be recommended for further thorough audiological assessment. If the participant passed following the rescreen, then the participant was judged as pass. The hearing screening was conducted in a quiet environment, with minimal visual and auditory distractions (i.e., ambient noise levels did not exceed recommended $49.5 \mathrm{~dB}$ SPL at $1000 \mathrm{~Hz}, 54.5 \mathrm{~dB}$ at $2000 \mathrm{~Hz}$, and 62 dB SPL at $4000 \mathrm{~Hz}$ when measured using a SLM). The audiometers used in the screening did meet ANSI S3.6-1989 and ISO 389-1991 standards. The audiometers used were also calibrated annually and daily check was performed prior to the screening to assure accuracy.

\section{Design}

This study is a correlational study, ${ }^{29}$ where no manipulation of a variable occurred; instead, data was gathered via screening to study the degree or relation between sets of scores on a speech, language, and hearing screening protocol using correlational statistics. It was a retrospective analysis, as a search was made using medical records that had been collected during a specific period of time previously. Screening data analyzed in this study was compiled during the years 2000 to 2010. Screening data was analyzed using correlational statistics to examine the presence of risk factors or evidence of different speech, language, and hearing disorders across children from different age-groups and genders.

Table I Frequencies and percentages of the children by name of the school and date of screening

\begin{tabular}{llll}
\hline Name of School & $\begin{array}{l}\text { Date of } \\
\text { Screening }\end{array}$ & Frequency & Percentage \\
\hline Al Falah School & 2000 & 185 & $12 \%$ \\
& 2001 & 195 & $13 \%$ \\
& 2002 & 195 & $13 \%$ \\
& 2006 & 85 & $6 \%$ \\
& 2007 & 192 & $12 \%$ \\
& 2008 & 192 & $12 \%$ \\
Al Manarat School & 2009 & 93 & $6 \%$ \\
Dar Al Hanan School & 2002 & 165 & $11 \%$ \\
& 2004 & 95 & $6 \%$ \\
Dar Al Bir Orphanage & 2007 & 49 & $3 \%$ \\
Jeddah Knowledge & 2008 & 29 & $2 \%$ \\
Total & 2003 & 23 & $1 \%$ \\
\hline
\end{tabular}

Table 2 Frequencies and percentages of the children according to the age intervals

\begin{tabular}{lll}
\hline Age Interval (Years.Months) & Frequency & Percentage \\
\hline Less than 4.7 & 2 & $0.10 \%$ \\
\hline $4.7-5.8$ & 9 & $0.60 \%$ \\
\hline $5.2-5.8$ & 225 & $14.60 \%$ \\
\hline $5.9-6.3$ & 950 & $61.60 \%$ \\
\hline $6.4-6.10$ & 357 & $23.10 \%$ \\
\hline Total & 1543 & $100.00 \%$ \\
\hline
\end{tabular}

Table 3 Reliability coefficients of the components of JISH school screening test

\begin{tabular}{ll}
\hline $\begin{array}{l}\text { Components of JISH School } \\
\text { Screening Test }\end{array}$ & Reliability Coefficients \\
\hline Hearing Screening Results & 0.92 \\
\hline Speech-Language Results & 0.94 \\
\hline Articulation of Speech & 0.9 \\
\hline Voice & 0.92 \\
\hline Fluency & 0.95 \\
\hline Hearing Loss & 0.96 \\
\hline Language Expression & 0.98 \\
\hline Language Comprehension & 0.96 \\
\hline
\end{tabular}

\section{Data analysis}

Several statistical tests and measurements were used for the data analysis. These included the following: the one-way analysis of variance ANOVA (F) test, used to determine the effect of age on the performance of the children and also to detect the differences of means among independent variables, ${ }^{30}$ the Pearson Correlation Coefficient, used to test the internal constant validity, samples differences were also calculated with the chi square test for independent samples $\left(\chi^{2}\right)$, $\mathrm{t}$-Test for Correlated Groups, used to determine the differences among participants, ${ }^{31}$ and finally the Cronbach's alpha, used to test scorer reliability scores. ${ }^{32}$

\section{Results and discussion}

Analysis of results showed statistically significant prevalence rates in all areas for which screening was conducted: hearing $(7.1 \%$ "0.0000"); language comprehension ( $1.7 \%$ "0.0000); language 
expression $(2.7 \%$ "0.0000); Voice $(5.1 \%$ " 0.0000$)$; Fluency $(3.2 \%$ " 0.0000$)$, and Articulation (30.3\%). The results are explained in depth in the following sections:

\section{Hearing screening section results}

One hundred fourteen of the 1543 children screened (7.4\%) failed the hearing screening for the frequencies tested. These results were significant at a significance level of $\leq 0.05$. (Table 4).

Table 4 Percentage and frequency of the hearing screening section

\begin{tabular}{lllll}
\hline Pass/Fail & Frequency & Percentage & $\begin{array}{l}\text { Chi- } \\
\text { square }\end{array}$ & Significance \\
\hline Pass & 1429 & 92.6 & 1120.69 & 0 \\
\hline Fail & 114 & 7.4 & & \\
\hline Total & 1453 & 100 & & \\
\hline
\end{tabular}

$P \leq 0.05$

\section{Articulation section results}

Four hundred and sixty eight children (30.3\%) exhibited articulation errors. The results of the Chi Square analysis revealed significant statistical difference. Table 5 below demonstrates that the Chi Square was $238.787(0.0000)$ at a significant level of $\leq 0.05$. Types of Articulation Errors:

Table 5 Percentage and frequency of the articulation skills section

\begin{tabular}{lllll}
\hline Pass/Fail & Frequency & Percentage & $\begin{array}{l}\text { Chi- } \\
\text { square }\end{array}$ & Significance \\
\hline Pass & 985 & 67.8 & 238.787 & 0 \\
\hline Fail & 468 & 32.2 & & \\
\hline Total & 1453 & 100 & & \\
\hline
\end{tabular}

$P \leq 0.05$

Due to a lack of research on the frequency of the Arabic articulation errors and the highly frequent misarticulated sounds in Arabic, an analysis was conducted to investigate this issue. A statistical analysis was conducted for the groups of the misarticulated sounds in all of the children screened. The results revealed that the fricative and emphatic fricative alveopalatal sounds $(/ \mathrm{s} /, / \mathrm{z} /, / \mathrm{s} /)$ were the highly frequent misarticulated sounds $(37.5 \%)$. The next group of highly affected sounds included the fricative, emphatic fricative, alveodental, pharyngeal and uvular sounds (/s/, /z/ /s/, /S/, /dZ/, /X/, / $\square /)$. These sounds occurred in the $16.5 \%$ of the $30.3 \%$ who failed the articulation section.

\section{Language comprehension section results}

Twenty six children (1.7\%) did not pass the language comprehension screening section. The results of the Chi Square analysis revealed significant statistical difference. Table 6 below demonstrates that the Chi Square was $1440.752(0.0000)$ at a significant level of $\leq 0.05$.

Table 6 Percentage and frequency of the language comprehension section

\begin{tabular}{lllll}
\hline Pass/Fail & Frequency & Percentage & $\begin{array}{l}\text { Chi- } \\
\text { square }\end{array}$ & Significance \\
\hline Pass & 1517 & 98.3 & 1440.752 & 0 \\
\hline Fail & 26 & 1.7 & & \\
\hline Total & 1453 & 100 & & \\
\hline
\end{tabular}

$P \leq 0.05$

\section{Language expression section results}

Forty one children $(2.7 \%)$ did not pass the language expression screening section. The results of the Chi Square analysis revealed significant statistical difference. Table 7 below demonstrates that the Chi Square was $1383.358(0.0000)$ at a significant level of $\leq 0.05$

Table 7 Percentage and frequency of the language expression section

\begin{tabular}{lllll}
\hline Pass/Fail & Frequency & Percentage & $\begin{array}{l}\text { Chi- } \\
\text { square }\end{array}$ & Significance \\
\hline Pass & 1502 & 97.3 & 1383.358 & 0 \\
\hline Fail & 41 & 2.7 & & \\
\hline Total & 1453 & 100 & & \\
\hline$P \leq 0.0$ & & & &
\end{tabular}

\section{Voice section results}

The results of the screenings indicated that 78 children exhibited voice problems $(5.1 \%)$. Table 8 revealed that the Chi Square was 1246.77. This result revealed a statistical difference $(0.0000)$ at a significant level of $\leq 0.05$.

Table 8 Percentage and frequency of the voice section

\begin{tabular}{lllll}
\hline Pass/Fail & Frequency & Percentage & $\begin{array}{l}\text { Chi- } \\
\text { square }\end{array}$ & Significance \\
\hline Pass & 1465 & 94.9 & 1246.77 & 0 \\
\hline Fail & 78 & 5.1 & & \\
\hline Total & 1453 & 100 & & \\
\hline$P \leq 0.05$ & & & &
\end{tabular}

\section{Fluency section results}

The prevalence of the fluency disorder was 3.2\% with a Chi-Square of 1353.224 and a statistical difference of 0.0000 at a significant level of $\leq 0.05$ (Table 9).

Table 10 below demonstrates that the analysis of all the screenings combined indicated that the total number of children screened (1543), 468 children failed the screening $(30.3 \%)$, with a Chi-square of 238.787 and a significant level of 0.0000 . This result is statistically significant at the level of $\leq 0.05$.

Table 9 Percentage and frequency of the fluency section

\begin{tabular}{lllll}
\hline Pass/Fail & Frequency & Percentage & $\begin{array}{l}\text { Chi- } \\
\text { square }\end{array}$ & Significance \\
\hline Pass & 1494 & 96.8 & 1353.224 & 0 \\
\hline Fail & 49 & 3.2 & & \\
\hline Total & 1453 & 100 & & \\
\hline
\end{tabular}

$P \leq 0.05$

\section{Gender differences}

The performance of the boys and girls was compared and analyzed using the two-way tailed ANOVA. The results are shown in Table 10 below.

The results shown in Table 10 above indicated statistical significance between the performance of the boys and the performance of the girls in the area of fluency, where the $0.18 \%$ of the boys exhibited a fluency disorder compared to a $0.10 \%$ of the girls $(0.006$ with std. Deviation .18344 for boys and 10127 for the girls).

The present study investigated the prevalence of speech, hearing and language disorders in Arabic speaking children in the Kingdom of Saudi Arabia. Due to the dearth of this data in the Arabic literature, the study attempted to provide various prevalence data to assist in the planning of services for children with such disorders. ${ }^{33}$ The analysis of the prevalence data obtained indicated that the prevalence of hearing 
problems was $7.4 \%$. This result supports previous studies suggesting that hearing loss is potentially a significant issue worldwide. Taha et al. ${ }^{34}$ for example, stated that hearing loss appears in two-thirds of individuals in developing countries in the world and the UN World Health Organization (WHO) reported that more than 25million people in Southeast Asia suffer from disabling hearing loss (http:// www.hear-it.org.dsp?area $=850$, retrieved June 20, 2016, 3;25
PM). ${ }^{35}$ In Saudi Arabia, high prevalence rates of hearing impairment might be also attributed to genetic factors and consanguinity. ${ }^{36}$ The current study supports the results of most of the hearing prevalence studies conducted in Saudi Arabia. Abolfotoh et al., ${ }^{37}$ for example, investigated the prevalence of hearing loss in 974 schoolboys between the ages of 6 and 12years in the city of Abha, Asir region, and found out a prevalence rate of $4.4 \%$.

Table 10 Two-way analysis of variance for gender

\begin{tabular}{|c|c|c|c|c|c|c|}
\hline Area of Screening & Gender & $\mathbf{N}$ & Mean & Standard Deviation & $\mathbf{t}$ & Sig. (2-Tailed) \\
\hline \multirow[t]{2}{*}{ Hearing Screening Result } & Male & 1349 & 0.92 & 0.268 & -1.8 & 0.06 \\
\hline & Female & 194 & 0.95 & 0.211 & & \\
\hline \multirow[t]{2}{*}{ Speech-Language Result } & Male & 1349 & 0.68 & 0.468 & -5.09 & 0 \\
\hline & Female & 194 & 0.83 & 0.377 & & \\
\hline \multirow[t]{2}{*}{ Voice Problems } & Male & 1349 & 0.0534 & 0.22486 & -1.617 & 0.107 \\
\hline & Female & 194 & 0.0309 & 0.17357 & & \\
\hline \multirow[t]{2}{*}{ Fluency Problems } & Male & 1349 & 0.0348 & 0.18344 & 2.781 & 0.006 \\
\hline & Female & 194 & 0.0103 & 0.10127 & & \\
\hline \multirow[t]{2}{*}{ Hearing Loss } & Male & 1349 & $0.074 I$ & 0.26208 & 1.657 & 0.09 \\
\hline & Female & 194 & 0.0464 & 0.21088 & & \\
\hline \multirow[t]{2}{*}{ Language Expression } & Male & 1349 & 0.0274 & 0.16339 & 0.61 & 0.542 \\
\hline & Female & 194 & 0.0206 & 0.14247 & & \\
\hline \multirow[t]{2}{*}{ Language Comprehension } & Male & 1349 & 0.0178 & 0.13224 & 0.922 & 0.357 \\
\hline & Female & 194 & 0.0103 & 0.10127 & & \\
\hline
\end{tabular}

$P \leq 0.05$

The analysis of the language results shows that $1.7 \%$ of the children manifested some difficulty in the area of language comprehension (receptive language), and $2.7 \%$ of the children had problems in the expressive language domain. It is evident that the prevalence in the area of receptive language comes as the lowest compared with all other speech and language results of the present study. This finding also confirms the findings of Keegstra et al., ${ }^{38}$ who stated that "problems with language production occurred more often than problems with language comprehension" (P. 817). In their study, Keegstra et al., ${ }^{39}$ investigated language problems in 240 Dutch children ( 174 boys and 66 girls) between the ages of 1.11 and 5.04 years. They found that $10 \%$ of the children exhibited language comprehension problems whereas $21 \%$ of the children had deficits in their expressive language.

The analysis of the stuttering data revealed an estimated prevalence of $3.2 \%$. This result indicates higher prevalence rate than the results reported by McKinnon et al. ${ }^{40}$ who reported a prevalence rate of $0.33 \%$ among Australian students (kindergarten students through grade 6). However, it should be noted that the McKinnon study incorporated several methods to obtain prevalence (e.g., direct testing, teachers' reports and parents' reports). Contrastingly, the results of the present study are closely similar with the prevalence rate reported by Proctor et al., ${ }^{41}$ In their study, the prevalence of stuttering in the African American (AA) children and in the European Children between the ages of 2 and 5years was investigated to determine if stuttering is overrepresented in the AA population. Parents, teachers and speechlanguage pathologists collected the data. Based on the data collected by teachers, the results showed that the prevalence of stuttering among the AA children was to be $2.51 \%$ and $1.70 \%$ among the European American children; however, the results of the investigators indicated that the prevalence of stuttering among the AA children was $2.60 \%$ and $2.44 \%$ among the European children. When combined together, the prevalence of stuttering was found to be $2.52 \%$, which is again close to the prevalence of stuttering among the Saudi children between the ages of 4:00 and 6:10 years. The comparison of the findings of the present study (3.2\%) with those reported by McLeod \& Harrison ${ }^{42}$ $(5.6 \%)$ revealed that stuttering disorder seems to be one of the mostly frequent universal prevalent speech disorders in the young children. Yet, it is important to note that the prevalence provided by McLeod \& Harrison ${ }^{43}$ is gleaned from the parents' reports on their children's speech skills. Direct assessment in their study was not conducted on speech skills. Considering further the surveys done in the last century, such as the one done by Mills and Streit in the academic year 1940-1941 in the schools of Holyoke, Massachusetts, where 4685 students were surveyed for speech problems, the results revealed a prevalence rate of $2.7 \%$ in students of young ages in grades one through grade three, i.e., between ages of 6 and 8years. This result agrees considerably with the findings of the present study, although both studies are different in terms of methodology and method of testing. The findings of the current study further confirm the prevalence rates reported by Andrews, Zebrowski ${ }^{4,45}$ who reported a prevalence rate of $4 \%$ to $5 \%$ in children aged between 2 and 4 years of age. The prevalence rate of voice disorder was found to be $5.1 \%$ in the present study. This rate is in direct agreement with the findings of previous studies and investigations. In the ASHA 2008 edition on Communication Facts, it was reported that "reported occurrence of hoarseness range from $6 \%$ to $23 \%$ in school-aged children.". ${ }^{46}$ The prevalence rate of the present study strongly supports the results reported by Baynes. ${ }^{47}$ One thousand and twelve students (512 boys and 500 girls) from the first, third, and sixth grades were surveyed over a three-month period for chronic hoarseness in the Willow Run Public School System of Willow Run, Michigan. The incidence was found to be $7.1 \%$. The survey also indicated that first graders exhibited the highest incidence ( $8 \%$ in boys and $9.2 \%$ in girls). Silverman \& $\mathrm{Zimmer}^{48}$ indicated that "the problem of hoarseness may be the most common childhood voice disorder" (P. 211). They conducted a voice screening for 162 children from kindergarten through eighth grade in the academic year 19721973. They found out that $23.4 \%$ of the 162 students had a chronic hoarseness voice problem. This percentage "appears strikingly high compared to that reported by Baynes $(7 \%)$ and by Pont $(9.1 \%){ }^{49}$ 
In conclusion, the findings of the present study are supportive evidence of the findings of most previous prevalence studies regarding hearing loss, voice disorders, fluency disorders, articulation problems, and receptive/expressive language delays. The results of this study are significant for the middle-eastern region, given the lack of prevalence studies in this region. The results call for professional and political attention and planning at all levels, particularly, by the decision makers in the Ministry of Education, Ministry of Health, and Ministry of Social Affairs. The results suggest that it would be of great value to mandate the initial screening of hearing, speech, and language skills of children prior to entering schools to make sure children possess adequate communication skills for learning and for academic success. These decisions become more critical given poor public awareness in the Arab region regarding the role of speech-language pathologists in helping children communicate better and thus achieve better academically, and the lack of speech and language therapy services in the public school arena, not only in the Kingdom of Saudi Arabia but throughout the Arab region. The results of the current study will potentially contribute to the significance of mandating the provision of speech-language therapy in the school settings in the Arab world.

\section{Conclusion}

The findings of the current study assure the need to screen speech, language and hearing skills of children before sending them to schools. This is significantly needed in the Middle East and in the whole Arab region due to the fact that general physical examinations are not enforced in most of the medical insurance policies, therefore, communication deficits will not be detected from early ages.

This retrospective study presents the results of school readiness screening conducted between the years of 2000 and 2010 at the Jeddah Institute for Speech and Hearing (JISH) in Jeddah, Saudi Arabia. The JISH Arabic Speech, Language, and Hearing Screening Protocol was individually administered by ASHA certified speech language pathologists to investigate the readiness of children prior to entering school and to identify children with speech, language, or hearing issues which may interfere with future academic performance and success in the first grade. A total of 1543 screening records (1349 male and 194 female) were reviewed and analyzed. Data was analyzed to obtain estimates of the prevalence of articulation errors, language expression and comprehension disorders, voice and fluency disorders, and hearing loss among children referred for screening. The data was further analyzed to identify the relation between gender and prevalence. Analysis showed statistically significant prevalence rates in all areas for which screening was conducted. Given the documented relationship between communication and academic skills, this study supports the need for mandated screening of hearing, speech, and language skills of all children prior to school enrollment.

Although the results of the current study agree with many other studies, these results should be interpreted with caution because of the following limitations. The results of the study might have been more accurate if the JISH Arabic Speech-Language-HearingScreening Protocol JISH protocol was standardized. The distribution of participants was not equal between boys and girls (1349 boys and 194 girls). More studies would be needed to investigate gender differences on school readiness. The other limitation of the current study might be related to the $80 \%$ accuracy pass/fail criterion for language comprehension and language expression subtests. If a $90 \%$ criterion was adopted instead, the results of the study would change and possibly more participants might have failed these subtests.

\section{Acknowledgments}

This study was funded by The Saudia Dairy and Foodstuff Co. (SADAFCO). The fund was provided to Jeddah Institute for Speech and Hearing (JISH) through the Corporate Social Responsibility Project between SADAFCO, Jeddah, Kingdom of Saudi Arabia, and JISH. This project aims to encourage research in the field of speechlanguage pathology in Arabic language and develop culturally and linguistically diagnostic and treatment materials in Arabic. The author thanks SADAFCO for their continued support and interest in our projects at JISH. I am also thankful to Dr. Susan Ainsleigh for taking the time for editing the whole article. A special thank you goes to mm. Sultana Alireza-Zahid, the JISH Chief Executive Director, for her continued support and encouragement.

\section{Conflicts of interest}

The researcher of the article has no personal or financial interest in SADAFCO Company which sponsored the development and standardization of JISH Arabic Speech-Language-Hearing-Screening Protocol JISH protocol.

\section{Funding}

None.

\section{References}

1. Bishop, DM Leonard, B. Speech and language impairments in children: causes, characteristics, intervention and outcome. Journal of Child Language. 2000;29(3):701-711.

2. Bender WA. Learning disabilities: Characteristics, identification, and teaching strategies. Needham Heights Allyn and Bacon, USA. 1998.

3. http://apps.who.int/iris/bitstream/10665/177094/1/9789241564960_ eng.pdf

4. McLeod S, Harrison LJ. Epidemiology of speech and language impairment in a nationally representative sample of 4-to 5-year-old children. J Speech Lang Hear Res. 2009;52(5):1213-1229.

5. US Preventive Services Task Force. Screening for speech and language delay in preschool children: Recommendation statement. Pediatrics. 2006;117(2):497-501.

6. Taha AA, Pratt SR, Farahat TM, et al. Prevalence and risk factors of hearing impairment among primary-school children in Shebin El-Kom District , Egypt. Am J Audio. 2010;19(1):46-60.

7. El-Zraigat A, Ibrahim. Hearing Impairment. Dar Wael for PrintingPublishing, Amman, Jordan. 2003.

8. Prevalence of hearing loss in Jordan. Alrai Daily Newspaper, Jordan. 2007. p. 21.

9. Beitchman JH, Nair R, Clegg M, et al. Prevalence of speech and language disorders in 5-year-old kindergarten children in the OttawaCarleton region. J Speech Hear Disord. 1986;51(2):98-110.

10. McLoughlin JA, Lewis, RB. Assessing students with special needs (6th edn), Englewood Cliffs, Prentice Hall, Inc. NJ, USA. 2001.

11. A L Keegstra, WA Knijff, W J Post, et al. Children with language problem in a speech and hearing clinic: background variables and extent of language problems. Int $J$ Pediatr Otorhinolaryngol. 2007;71(5):815-821.

12. McLoughlin JA, Lewis RB. Assessing students with special needs (6th edn), Englewood Cliffs, Prentice Hall, Inc., NJ, USA. 2001.

13. Beitchman JH, Nair R, Clegg M, et al. Prevalence of speech and language disorders in 5-year-old kindergarten children in the OttawaCarleton region. J Speech Hear Disord. 1986;51(2):98-110. 
14. Fundudis, T Kolvin, L Garside RF. Speech retarded and deaf children: The psychological development. Academic Press, London, UK. 1979.

15. Tumoi S, Ivanoff P. Incidence of speech and hearing disorders among kindergarten and grade one children. Special Education in Canada. 1977;51:5-8.

16. Stevenson J, Richman N. The prevalence of language delay in a population of three-year-old children and its association with general retardation. Dev Med Child Neurol. 1976;18(4):431-441.

17. Peckham CS. Speech defects in a national sample of children aged seven years. British Journal of Disorders ofCommunication. 1973;8(1):2-8.

18. Hull FM, Mielke PW, Timmons RJ, et al. The national speech and hearing survey: Preliminary results. ASHA. 1971;13(9):501-509.

19. Pronovost W. A survey of services for the speech and hearing handicapped in New England. Journal of Speech and Hearing Disorders. 1951;16:148-156

20. Alice W Mills, Helen Streit. Report of a speech survey, Holyoke, Massachusetts. Journal of Speech Disorders. 1942;7:161-167.

21. Beitchman JH, Nair R, Clegg M, et al. Prevalence of speech and language disorders in 5-year-old kindergarten children in the OttawaCarleton region. J Speech Hear Disord. 1986;51(2):98-110.

22. Heidi D Nelson, Peggy Nygren, Miranda Walker, et al. Screening for speech and language delay in preschool children: Systematic evidence review for the US Preventive Services Tasks Force. Pediatrics. 2006;117(2):e298-e319.

23. http://www.jish.org

24. Fluharty N Buono. Fluharty Preschool Speech and Language Screening Test. Austin, Texas, USA. 2001

25. McLoughlin JA, Lewis RB. Assessing students with special needs (6th edn), Englewood Cliffs, Prentice Hall, Inc., NJ, USA. 2001.

26. Pierangelo R, Giuliani GA. Assessment in special education: A practical approach. Allyn \& Bacon, Boston, MA, USA. 2002.

27. www.ASHA.org

28. Ballachanda BB, Roeser RJ, Kemp RJ. Control and prevention of disease transmission in audiology practice. American Journal of Audiology. 1996;5(1):74-82.

29. Creswell JW. Research design: Qualitative, Quantitative, and Mixed Method Approaches, Sage Publications, USA. 2009.

30. Crowl T. Fundamentals of education research (2nd edn), Madison, WI: Brawn \& Benchmark Publishers, USA. 1990.

31. Schiavetti N, Metz DE. Evaluating research in communicative disorders (3rd edn), Needham Heights, Allyn \& Bacon, MA, USA. 1997.

32. Cortina JM. What is Coefficient Alpha? An Examination of Theory and Applications. Journal of Applied Psychology. 1993;(78):98-104.
33. McKinnon DH, McLeod S, Reilly S. The prevalence of stuttering, voice, and speech- sound disorders in primary school students in Australia. Lang Speech Hear Serv Sch. 2007;38(1):5-15.

34. Taha AA, Pratt SR, Farahat TM, et al. Prevalence and risk factors of hearing impairment among primary-school children in Shebin El-Kom District , Egypt. Am J Audiol. 2010;19:46-60.

35. Low Public Awareness of Hearing Loss. 2011.

36. El Mouzan MI, Al Salloun AA, Al Herbish AS, et al. Consanguinity and major genetic disorders in Saudi Children: A community-based crosssectional study. Ann Saudi Med. 2008;28(3):169-173.

37. Abolfotouh MA, Ghieth MM, Badawi IA. Hearing Loss and other ear problems among schoolboys in Abha, Saudi Arabia. Ann Saudi Med. 1995;15(4):423-426.

38. Keegstra AL, Knijff WA, Post WJ, et al. Children with language problem in a speech and hearing clinic: background variables and extent of language problems. Int J Pediatr Otorhinolaryngol. 2007;71(5):815-821.

39. Keegstra AL, Knijff WA, Post WJ, et al. Children with language problem in a speech and hearing clinic: background variables and extent of language problems. Int J Pediatr Otorhinolaryngol. 2007;71(5):815-821.

40. McKinnon DH, McLeod S, Reilly S. The prevalence of stuttering, voice, and speech- sound disorders in primary school students in Australia. Lang Speech Hear Serv Sch . 2007;38(1):5-15.

41. Proctor A, Yairi E, Duff MC, et al. Prevalence of stuttering in African American preschoolers. Journal of Speech, Language, and Hearing Research. 2008;51:1465-1479.

42. McLeod S, Harrison LJ. Epidemiology of speech and language impairment in a nationally representative sample of 4- to 5 -year-old children. J Speech Lang Hear Res . 2009;52(5):1213-1229.

43. McLeod S, Harrison LJ. Epidemiology of speech and language impairment in a nationally representative sample of 4- to 5 -year-old children. J Speech Lang Hear Res . 2009;52(5):1213-1229.

44. Andrews G. The epidemiology of stuttering In: Curlee \& Perkins (Eds.), Nature and treatment of stuttering: New directions. College-Hill Press, San Diego, CA, USA. 1984

45. Zebrowski PM. Developmental Stuttering. Pediatric Annals. 2003;32(7):453-458.

46. Communication Facts: Special population: literacy. 2011.

47. Baynes AR. An incidence study of chronic hoarseness among children. $J$ Speech Hear Disord. 1966;31(2):172-176.

48. Silverman EM, Zimmer CH. Incidence of chronic hoarseness among school-age children. J Speech Hear Disord. 1975;40(2):211-215.

49. Silverman EM, Zimmer CH. Incidence of chronic hoarseness among school-age children. J Speech Hear Disord. 1975;40(2):211-215. 